Blob properties in full-turbulence simulations of the TCV scrape-off layer

This content has been downloaded from IOPscience. Please scroll down to see the full text.

2017 Plasma Phys. Control. Fusion 59055009

(http://iopscience.iop.org/0741-3335/59/5/055009)

View the table of contents for this issue, or go to the journal homepage for more

Download details:

IP Address: 128.179.255.79

This content was downloaded on 07/05/2017 at 07:05

Please note that terms and conditions apply.

You may also be interested in:

Blob structure and motion in the edge and SOL of NSTX

S J Zweben, J R Myra, W M Davis et al.

Numerical simulations of blobs with ion dynamics

A H Nielsen, J Juul Rasmussen, J Madsen et al.

Drive of parallel flows by turbulence and large-scale $\mathrm{E} \times \mathrm{B}$ transverse transport in divertor geometry

D. Galassi, P. Tamain, H. Bufferand et al.

Characterization of the blob generation region and blobby transport in a stellarator

G Fuchert, G Birkenmeier, M Ramisch et al.

Experimental and numerical characterization of the turbulence in the scrape-off layer of MAST

F Militello, $\mathrm{P}$ Tamain, W Fundamenski et al.

A comparison between a refined two-point model for the limited tokamak SOL and self-consistent plasma turbulence simulations

C Wersal, P Ricci and J Loizu

Hermes: global plasma edge fluid turbulence simulations

B D Dudson and J Leddy

The influence of plasma edge dynamics on blob properties in the stellarator TJ-K

G Fuchert, G Birkenmeier, B Nold et al.

The structure and poloidal dynamics of blob filaments in TJ-K

S Garland, G Fuchert, M Ramisch et al. 


\title{
Blob properties in full-turbulence simulations of the TCV scrape-off layer
}

\author{
F Nespoli, I Furno, B Labit, P Ricci, F Avino, F D Halpern ${ }^{1}$, F Musil and \\ F Riva
}

Ecole Polytechnique Fédérale de Lausanne (EPFL), Swiss Plasma Center (SPC), CH-1015 Lausanne, Switzerland

E-mail: nespolifederico@gmail.com

Received 9 September 2016, revised 10 February 2017

Accepted for publication 23 February 2017

Published 21 March 2017

\begin{abstract}
To investigate blob properties in the tokamak scrape-off layer (SOL), we perform dedicated numerical nonlinear simulations of plasma turbulence in the SOL of a TCV discharge using the Global Braginskii Solver code. A blob detection technique is used for the first time in a threedimensional (3D) full-turbulence simulation to track the motion of the filaments in the SOL. The specific size, density amplitude and radial velocity of the blobs are computed, with the typical values being $7.4 \rho_{s}, 0.33 n_{e}$ and $0.016 c_{s}$, respectively. The analysis of blob structure in the parallel direction shows that the blobs are partially detached from the limiter. The cross correlation analysis shows how the blobs are born all along the entire field line, not being generated primarily on the low field side SOL and expanding towards the limiter. The blob radial velocity agrees well with the inertial branch of the existing scaling law. The radial particle and heat fluxes given by blobs are shown to be responsible of up to $100 \%$ and $70 \%$ of the turbulent particle and heat flux in the far SOL, respectively. The results of a second simulation with a 40 times higher resistivity are also discussed.
\end{abstract}

Keywords: scrape-off layer, blob, tokamak, TCV, GBS, simulation, turbulence

(Some figures may appear in colour only in the online journal)

\section{Introduction}

Plasma filaments (blobs) are an ubiquitous feature of plasmas in open magnetic field lines and are routinely detected in the tokamak scrape-off layer (SOL). Due to their radially outwards motion, they are believed to enhance the cross field transport in the SOL $[1,2]$ with respect to purely collisional transport in the absence of turbulence, contributing substantially to the heat deposition on the first wall and divertor plates, both on the high field side (HFS) and on the low field side (LFS). The comprehension of their dynamics is hence of crucial importance for a better prediction of the heat loads onto the first wall. Blob dynamics has been investigated experimentally in tokamaks [3-6] and in basic plasmas experiments [7-13]. Though, blob measurements in tokamaks with an exhaustive diagnostic coverage, as in basic plasmas

\footnotetext{
1 Present address: General Atomics, P.O. Box 85608, San Diego, California 92186-9784, United States of America.
}

experiments [7], is currently prohibitive. Furthermore, the mechanisms governing blob formation and dynamics could differ in basic plasma experiments and in tokamaks SOL.

Blobs dynamics has also been investigated with numerical simulations of single seeded blobs [14-17], which do not give any indication on blob shape and behavior in a SOL plasma, since they are missing the interaction with a $3 \mathrm{D}$, turbulent background plasma.

Blob tracking techniques have been previously employed to investigate blob dynamics in basic plasma experiments $[7,9]$, on gas-puff imaging data of the tokamak SOL [3, 18], and on two-dimensional (2D) simulations [19]. To have a better understanding of blobs generation and dynamics, exploiting the advantages of a full coverage diagnostic on a realistic SOL plasma, we apply for the first time a blob detection technique to a full-turbulence numerical 3D simulation of the entire SOL of a plasma discharge. The SOL of a TCV inboard-limited L-mode plasma is modeled using the Global Braginskii Solver (GBS) code [20, 21]. The blobs, 
generated self-consistently by the SOL plasma turbulence, are detected and tracked in time, from their birth to their death.

This paper is structured as follows: in section 2 we describe the numerical simulations of the TCV SOL using the GBS code. In section 3, the blob detection and tracking method is described. In section 4 , the blob birth position is discussed, while in section 5 , the $3 \mathrm{D}$ shape of the blobs is analyzed. In section 6 , the results of the cross-correlation analysis are exposed, confirming the results from the previous section and giving some more insight on blob generation. In section 7 , the velocities resulting from blob tracking are analyzed and compared with an existing scaling law. Finally, in section 8 we quantify the contribution of the blobs to the radial particle and heat fluxes.

\section{Nonlinear simulations with the GBS code}

We perform dedicated numerical nonlinear simulations using the GBS code [20, 21]. By solving the drift-reduced Braginskii equations, GBS allows for the self-consistent description of equilibrium (time-averaged) and fluctuating quantities in 3D over the entire volume of the SOL. Effects due to finite aspect ratio, ion temperature and magnetic shear are included in the simulations, to have a more realistic description of the TCV SOL. Also, finite ion temperature is considered important for blob dynamics [22] and may indeed play a role in the TCV SOL, where ions and electrons have similar temperatures. The equations determining the plasma dynamics are detailed in $[21,23]$. The boundary conditions at the limiter are described in [24]. At the inner and outer radial boundaries of the simulations, Neumann boundary conditions are used for all quantities, with the exception of the plasma potential (at the outer boundary) and the vorticity (at both boundaries), for which Dirichlet conditions are imposed. These simulations feature only open field lines and plasma density and temperature (ion and electron) sources mimic the injection of plasma from the core into the SOL, building up the profiles over time, which in turn generate the turbulence. The flux surface on which the sources are located corresponds hence to the last closed flux surface (LCFS) of the real tokamak plasma.

In this paper, the results of two simulations are discussed: in the first one (A), the SOL of a TCV discharge is modeled. It is a circular inboard limited ohmic L-mode deuterium plasma, with plasma current and toroidal magnetic field on axis being $I_{p}=145 \mathrm{kA}$ and $B=1.45 \mathrm{~T}$, respectively. The values of the plasma density and temperature at the LCFS, $n_{e 0}=5 \times$ $10^{18} \mathrm{~m}^{-3}$ and $T_{e 0}=25 \mathrm{eV}$, are deduced from Langmuir probes embedded in the limiter. They set the normalized Spitzer resistivity $\nu=q_{e} n_{e 0} R_{0} /\left(m_{i} c_{s 0} \sigma_{\|}\right) \propto n_{e 0} R_{0} \Lambda m_{e} /\left(m_{i} c_{s 0} T_{e 0}^{3 / 2}\right)$, $\nu=5.9 \times 10^{-4}$, and the dimensionless size of the system through the ion sound Larmor radius $\rho_{s}=m_{i} c_{s 0} /\left(q_{e} B\right)=$ $0.5 \mathrm{~mm}$, where $\Lambda=15$ is the Coulomb logarithm, $R_{0}=$ $0.84 \mathrm{~m}$ is the major radius of the plasma, $c_{s 0}$ is the ion sound speed based on the electron temperature at the LCFS, $T_{e 0}$, and $m_{i}, m_{e}$ and $q_{e}$ are the ion mass, electron mass, and electron charge, respectively. The resulting simulation domain consists of $128 \times 820 \times 128$ points in the radial $(x)$, poloidal $(y)$ and toroidal $(z)$ direction, respectively. The sources of plasma temperature and density are located at $x=26$. The shape of the sources is gaussian in the radial direction with a width of 4 grid points. The sources are poloidally and toroidally uniform. In the following, we will consider $x_{\mathrm{LCFS}}=32$ as the position of the LCFS. The safety factor $q=3.2$, the magnetic shear $\hat{s}=1.5$ and the inverse aspect ratio $\epsilon=0.24$ are obtained from the magnetic reconstruction of the discharge, provided by the LIUQE code [26]. The ion temperature at the LCFS is assumed to be $T_{i 0}=T_{e 0}$, since no ion temperature measurement was available for that discharge. Resistivity can affect substantially blobs dynamics by electrically disconnecting from the target sheaths and subsequently increasing their convective velocity, as predicted in [25] and experimentally measured in [4, 27, 28]. The influence of resistivity on blob dynamics has been investigated with seeded simulations in [17, 29]. To investigate the role of resistivity on blob dynamics, we perform a second simulation (B) identical to the first one, except for the resistivity, which is 40 times larger. The two numerical simulations provide, for every frame, the $3 \mathrm{D}$ distribution of the plasma density $n$ (quasi neutrality is assumed), the plasma potential $\phi_{p}$, the electron and ion temperature $T_{e}$ and $T_{i}$, the electron and ion parallel velocities $v_{\|, e}$ and $v_{\|, i}$. Our analysis is performed over a turbulent quasisteady state, where plasma injection from the sources, turbulent transport and losses to the limiter balance each other. The quasi-steady state, time-averaged quantities for the two simulations are described in details in [30]. The analysis presented in this work is applied to a simulated time interval of 23 $R_{0} / c_{s 0}$ corresponding to $0.55 \mathrm{~ms}$, the results output frequency being $3 \mathrm{MHz}$. The simulation output result at a given time will be referred to in the following as 'frame'. A snapshot of the normalized plasma density in a poloidal plane of simulation $\mathrm{A}$ is shown in figure 1(a), together with the limiter geometry in the simulation (thick red) and TCV (dashed red) respectively. The set of coordinates that is used in the following is also displayed: $\theta$ is the poloidal angle and $r_{u}$ is the upstream radial coordinate, with $r_{u}=0$ at the LCFS. The relationship between the grid coordinates $(x, y)$ and the coordinates $\left(r_{u}, \theta\right)$ is $r_{u}=\left(x-x_{\mathrm{LCFS}}\right) \cdot 0.78 \rho_{s}, \theta=2 \arctan \left(\sqrt{\frac{1+\epsilon}{1-\epsilon}} \tan \theta^{*}\right)$, where $\theta^{*}=\left(y-n_{y} / 2\right) 2 \pi / n_{y}$, with $n_{y}=820$ the number of grid points in the poloidal direction. As discussed in [30], the simulated heat flux profiles agree quantitatively with the experimental ones [31], showing the presence of two distinct regions in the SOL, that can therefore be separated into 'near' and 'far' SOL. The near SOL, extending a few millimeters from the LCFS, is characterized by steep gradients of plasma temperature and density and is of crucial importance for peak heat loads on the tokamaks first wall [32]. The far SOL, characterized by flatter profiles, is typically a few centimeters wide and is at the origin of the main heat loss channel onto the first wall.

\section{Blob detection technique}

A pattern recognition technique, implemented on the basis of the one used in [7] on experimental data, is employed for the first time on full-turbulence $3 \mathrm{D}$ simulations to track the 
a)

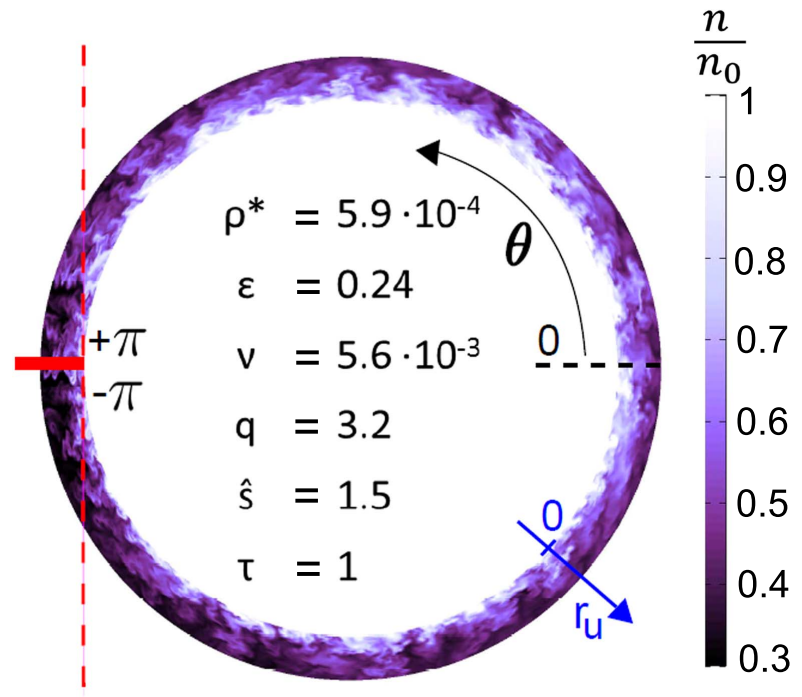

b)

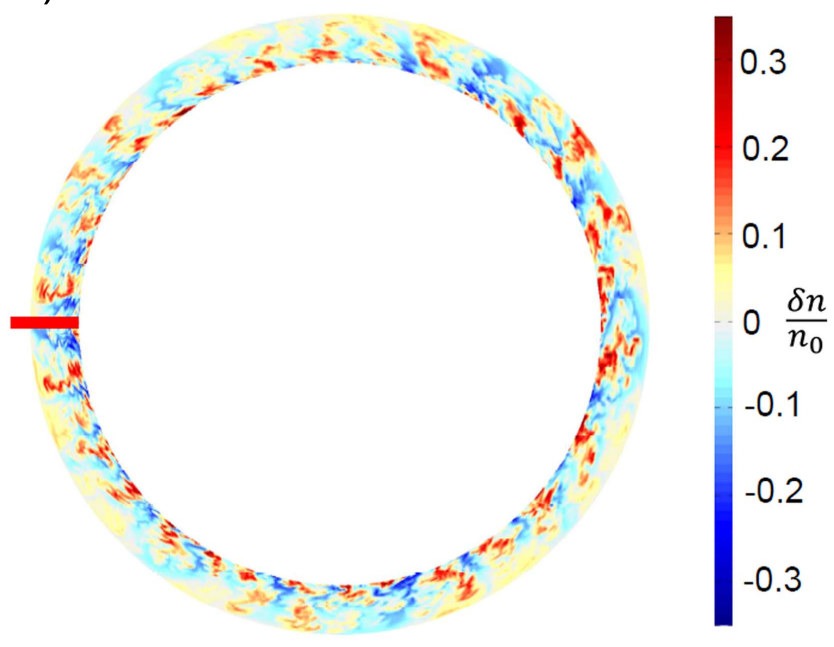

Figure 1. (a) Snapshot of normalized plasma density $n / n_{0}$ from simulation A. The coordinate system $\left(r_{u}, \theta\right)$ is shown. The limiter geometry for TCV and for the simulation are depicted with a dashed red line and a thick red line, respectively. The simulation parameters are displayed: normalized ion sound Larmor radius $\rho^{*}=\rho_{s} / R$, inverse aspect ratio $\epsilon$, Spitzer resistivity $\nu$, safety factor $q$, magnetic shear $\hat{s}$ and ion to electron temperature ratio $\tau=T_{i, 0} / T_{e 0}$. (b) Snapshot of normalized plasma density fluctuation $\delta n / n_{0}$.

motion of the filaments and to study their dynamics. The condition for the blob detection is $n\left(r_{u}, \theta, t\right)>\bar{n}\left(r_{u}, \theta\right)+$ $2.5 n_{\text {rms }}\left(r_{u}, \theta\right)$, where $\bar{n}$ and $n_{\text {rms }}$ are the local toroidally and time-averaged density and its standard deviation, respectively. The different blobs are identified in a given (2D) poloidal plane for every frame of the simulation through pattern recognition. They are then tracked to determine their radial and poloidal velocity $v_{r}, v_{\theta}$ : two structures on consecutive frames $b_{i}\left(t_{k-1}\right)$ and $b_{j}\left(t_{k}\right)$ are considered to be the same blob if the area $A$ of their intersection is larger than $10 \%$ of the area of the structure in the earlier frame: $A\left(b_{i}\left(t_{k-1}\right) \cap b_{j}\left(t_{k}\right)\right)>$ $A\left(b_{i}\left(t_{k-1}\right)\right) / 10$. Using the same intersection criterion, the algorithm is able to differentiate splitting and merging events. In particular, a splitting event occurs when a single blob in frame $t_{k-1}$ partially superimposes with two (or more) blobs in frame $t_{k}$. In this case, the previous blob trajectory is considered as terminated and two (or more) new blobs are born. A merging event occurs when two (or more) blobs in frame $t_{k-1}$ partially superimpose with one single blob in frame $t_{k}$. In this case, the previous blobs trajectories are considered as terminated and one new blob is born. Blobs living less than 6 frames $=0.08 R_{0} / c_{s 0}=2 \mu$ s are discarded from the analysis.

No restriction is imposed on the detected blob size at this stage, so the detection of blobs constituted only by one pixel (corresponding to the peak of the density fluctuation) is possible. Indeed, once the peak density fluctuation of the blob is detected, the blob size is determined a posteriori, with the procedure detailed in section 5 . Nevertheless, it is unlikely that such small blobs live at least the 6 time frames necessary for being kept for the rest of the analysis. No further assumptions are made on the field-alignment and toroidal extension of the detected blobs. Therefore, the same blob might be detected at several locations in the reference poloidal plane.

The blob velocity $\mathbf{v}$ is computed for each frame $t_{k}$ of its trajectory as the velocity of the center of mass of the blob $\mathbf{x}_{C M}$ using finite centered differences, i.e. $\mathbf{v}\left(t_{k}\right)=\left[\mathbf{x}_{C M}\left(t_{k+1}\right)-\right.$ $\left.\mathbf{x}_{C M}\left(t_{k-1}\right)\right] /\left(t_{k+1}-t_{k-1}\right)$. Therefore the velocity $\mathbf{v}$ is not defined for the first and the last frame for each trajectory.

Since the presented simulations are global 3D, all poloidal planes are equivalent from the statistical point of view. Therefore, the blob detection technique is applied to a single poloidal plane $(z=1$, corresponding to the toroidal angle $\phi=0$ ) of the simulation output, resulting in the detection and tracking of over 5900 blobs for simulation A. A typical result of the blob tracking is shown in figure 2 , in which the contour of a detected blob is displayed for subsequent frames. The results of the blob detection algorithm are summarized in table 1, where all the quantities, except from the blob life time $t_{l}$, are averaged over the blob trajectory, and the brackets denote ensemble averaging. The higher resistivity in simulation $\mathrm{B}$ results in the detection of $\sim 30 \%$ more filaments, consistently with the increase of the skewness of the density fluctuations reported in [30]. The blobs originating from merging events account for the $23 \%$ (22\%) of the total, while $24 \%$ (30\%) of the blobs results from splitting events, for simulations A (B), respectively. The average life time of the blobs is $\left\langle t_{l}\right\rangle=0.14 R_{0} / c_{s 0}=3.5 \mu \mathrm{s}$ for simulation $\mathrm{A}$ and $\left\langle t_{l}\right\rangle=0.16 R_{0} / c_{s 0}=4 \mu \mathrm{s}$ for simulation B.

By lowering the detection threshold from $2.5 n_{\mathrm{rms}}$ to $1.5 n_{\mathrm{rms}}$, the number of detected blobs increases by $50 \%$. Nevertheless, the statistical properties of the blobs, discussed in the following, do not vary substantially.

\section{Blob birth location}

In the following we discuss the blob birth spatial distribution resulting from the blob detection algorithm, and not the blob generation mechanism, which is beyond the scope of the present paper. In figures 3(a), (b) the histograms of birth events against the radial coordinate are shown for both 

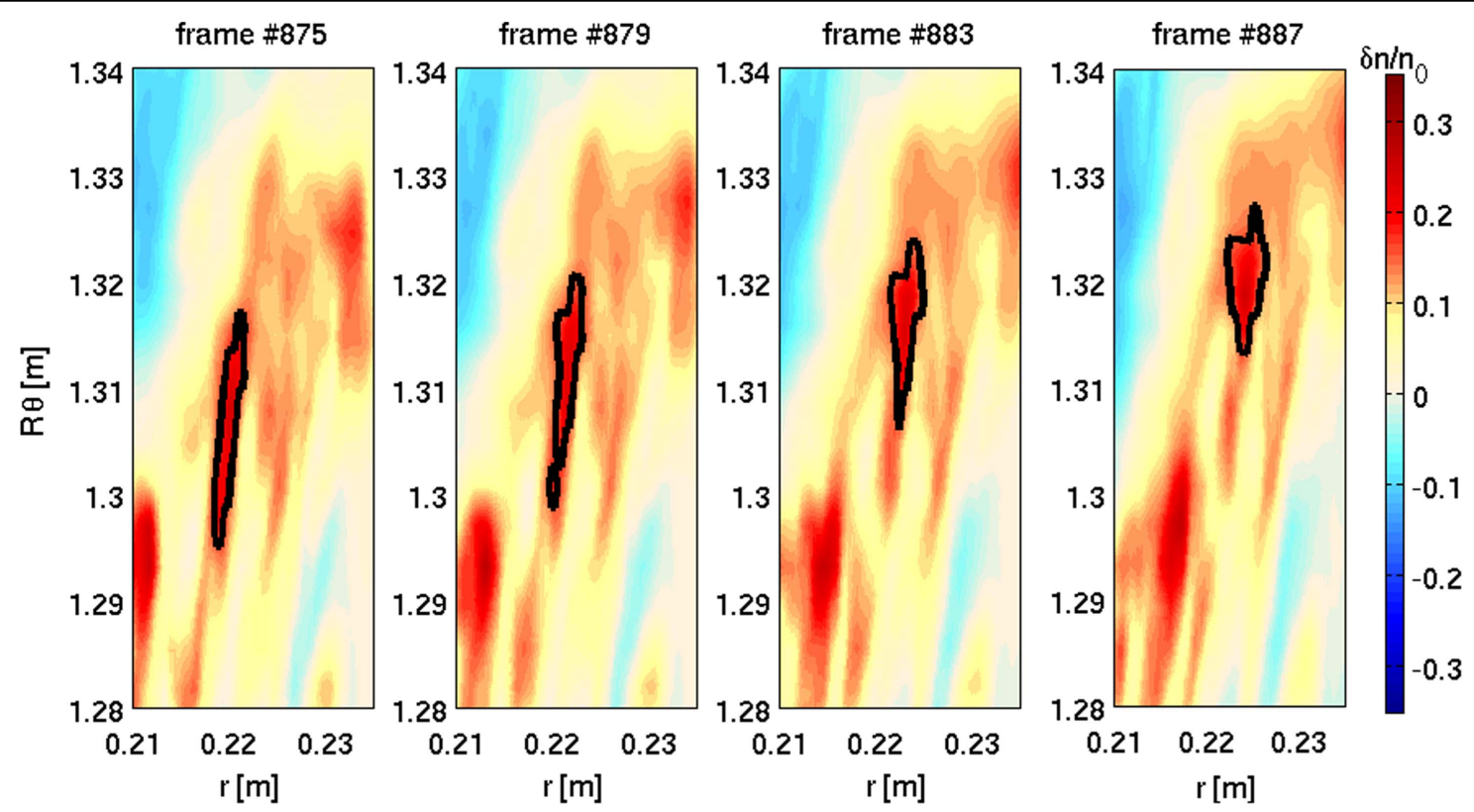

Figure 2. The contour of a blob, as detected by the pattern recognition algorithm, is plotted in black together with the normalized density fluctuation $\delta n / n_{0}$ in the poloidal plane, being $r$ and $R$ the plasma minor and major radius coordinates respectively, for 4 subsequent simulation frames (equally spaced by 4 frames, corresponding to $1.3 \mu \mathrm{s}$ ). Even though several blobs are present in the same simulation frame $(\sim 40)$, only one is plotted here for clarity.

Table 1. Summary of the results from the blob detection method for both simulations. The quantities are defined in the text.

\begin{tabular}{lcccccc}
\hline Simulation & \# Blobs & Merging & Splitting & $\left\langle a_{\theta}\right\rangle$ & $\left\langle a_{r}\right\rangle$ & $\left\langle a_{\|}\right\rangle$ \\
\hline $\mathrm{A}(1 \times \nu)$ & 5902 & $23 \%$ & $24 \%$ & $7.4 \rho_{s}$ & $4.5 \rho_{s}$ & $6026 \rho_{s}$ \\
$\mathrm{~B}(40 \times \nu)$ & 7611 & $22 \%$ & $30 \%$ & $6.3 \rho_{s}$ & $4.3 \rho_{s}$ & $4460 \rho_{s}$ \\
\hline Simulation & $\left\langle\delta n_{\max } / n_{\max }\right\rangle$ & $\left\langle t_{l}\right\rangle$ & $\left\langle\sigma_{l, \text { bottom }}\right\rangle$ & $\left\langle\sigma_{l, \text { top }}\right\rangle$ & $\left\langle v_{\theta}\right\rangle$ & $\left\langle v_{r}\right\rangle$ \\
\hline $\mathrm{A}(1 \times \nu)$ & 0.33 & $0.14 R_{0} / c_{s 0}$ & 0.35 & 0.30 & $0.011 c_{s 0}$ & $0.015 c_{s 0}$ \\
$\mathrm{~B}(40 \times \nu)$ & 0.30 & $0.16 R_{0} / c_{s 0}$ & 0.16 & 0.28 & $0.004 c_{s 0}$ & $0.020 c_{s 0}$ \\
\hline
\end{tabular}

simulations. From the detection algorithm, the birth of a blob can be due to three different mechanisms: (1) a blob is detected for the first time, i.e. it does not superimpose to any blob present in the previous simulation frame (2) two (or more) new blobs are born from the splitting of a single blob in the previous frame into two (or more) distinct parts (3) a blob is born from the merging of two or more blobs in the previous frame. The histogram of the blob birth position is dominated by the blobs that are detected for the first time (case 1), accounting for $\sim 50 \%$ of the total detected blobs for both simulations. The profiles of the birth rate are hence dominated by the 'first detected' blobs (red dashed lines in figure 3 ). The radial profile of the blob first detections is quite broad and peaks around $r_{u}=6 \mathrm{~mm}$ for simulation A. Increasing the resistivity, the point of maximum birth rate moves radially inwards to $r_{u}=3 \mathrm{~mm}$ and the profile is more pronounced. Also, as shown in figures 3(c), (d), where the histograms of birth events against the poloidal coordinate are shown for both simulations, the blobs are more likely to be first detected at the HFS SOL for simulation A and at the LFS SOL for simulation $\mathrm{B}$. This is consistent with the transport being more ballooned at higher resistivity [33]. The increase of the detection rate at the HFS in simulation A might be caused by a local increase of the $\mathbf{E} \times \mathbf{B}$ shearing rate.

\section{Characterization of the blob size and shape}

Following [9], we introduce here the typical blob size [34] and velocity [35]:

$$
a^{*}=\left(\frac{4 L^{2}}{\rho_{s} R_{0}}\right)^{1 / 5} \rho_{s}, \quad v^{*}=\left(\frac{2 L \rho_{s}^{2}}{R_{0}{ }^{3}}\right)^{1 / 5} c_{s 0},
$$

where $L=2 \pi q R_{0}$ and $R_{0}$ are the connection length and major radius respectively.

The linear size of each blob in the radial and poloidal direction, $a_{r}$ and $a_{\theta}$, are computed as the HWHM of the density fluctuation $\delta n\left(r_{u}, \theta, t\right)=n\left(r_{u}, \theta, t\right)-\bar{n}\left(r_{u}, \theta\right)$. The procedure to compute the radial HWHM is described in the following; in the poloidal and parallel direction a similar method is applied. Given a blob $b$, its density fluctuation at time $t$ is maximum at the point $\left(r_{b}, \theta_{b}\right)$, being $\delta n_{b, \max }=\delta n\left(r_{b}, \theta_{b}\right)$. The 

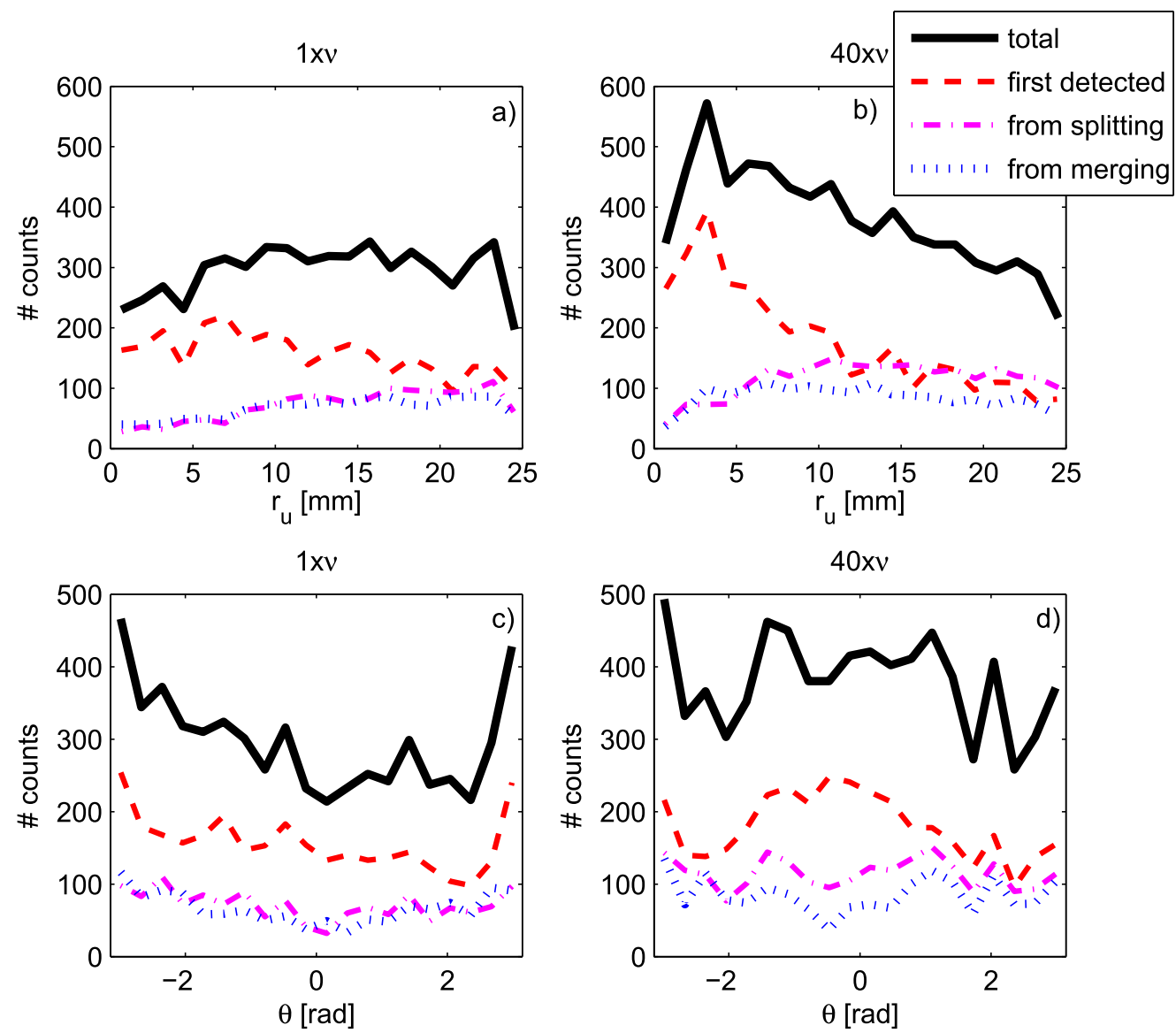

Figure 3. Histograms of the birth events along the radial coordinate $r_{u}$ (a), (b) and along the poloidal coordinate $\theta$ (c), (d), for simulation A (a), (c) and B (b), (d). The contribution to the total birth rate (thick black lines) of blobs originating from splitting (dash-dotted magenta) or merging events (dotted blue) is distinguished from the blobs that are detected for the first time (dashed red).

radial density fluctuation profile at the blob poloidal location $\delta n\left(r_{u}, \theta_{b}\right)$ is checked for intersections with the detected areas of all the other blobs in the same simulation frame. The part of the profile not superimposed to any other blob $\delta n^{\prime}\left(r_{u}, \theta_{b}\right)$ is extracted. The radial HWHM is then half of the extent of the region for which $\delta n^{\prime}\left(r_{u}, \theta_{b}\right)>\delta n_{b, \max } / 2$. The same computation is performed even for possible cases for which the reduced fluctuation profile $\delta n^{\prime}\left(r_{u}, \theta_{b}\right)$, due to intersection of the blob with other blobs on the same simulation frame, or with the limiter plate, remains always larger than $\delta n_{b, \max } / 2$. The average poloidal size of the filaments is $\left\langle a_{\theta}\right\rangle=7.4 \rho_{s}$ and $6.3 \rho_{s}$ for simulations A and B, respectively. The Fourier spatial transform of the density fluctuations shows a mode with a poloidal number $m=30$ extending from the LCFS up to $r_{u} \sim 10 \mathrm{~mm}$. This mode is visible in figure $1(\mathrm{~b})$, where a snapshot of the normalized density fluctuation $\delta n / n_{0}$ is shown. The average poloidal size of the blobs is comparable to the poloidal HWHM of the part of the mode with a positive density fluctuation, $L_{\theta, \mathrm{HWHM}} \sim L_{\theta} / 6=\left(2 \pi a_{\min } / \mathrm{m}\right) / 6=14 \rho_{\mathrm{s}}$.

The blobs are found to be elongated in the poloidal direction being $a_{r} / a_{\theta} \sim 1 / 2$, as displayed in figure 4 , where the joint probability distribution of the poloidal and radial blob size (normalized to the typical blob size $a^{*}$ ) is shown for both simulations.

Taking advantage of the 3D geometry of the simulations, the density profile along the field line is extracted for blobs detected in a given 2D poloidal plane. From this profile, the maximum blob (normalized) density fluctuation along the field line $\delta n_{\max } / n_{\max }$, the blob size in the parallel direction $a_{\|}$ and the pre-sheath density drop along the field line $\sigma_{l}=\delta n_{\text {limiter }} / \delta n_{\max }$ are determined, where $n_{\max }$ and $\delta n_{\max }$ are the maximum of the density and the corresponding density fluctuation in the parallel profile, and $\delta n_{\text {limiter }}$ is the density fluctuation at the limiter, along the same field line. Similarly to $a_{\theta}$ and $a_{r}, a_{\|}$is computed as the HWHM of the fluctuation profile along the field line, $\delta n\left(r_{u, b}, s, t\right)=n\left(r_{u, b}, s, t\right)-$ $\bar{n}\left(r_{u, b}, s\right)$, where $s$ is the coordinate along the field line and $r_{u, b}$ is the radial position of the detected blob in the reference poloidal plane. The maximum of the density fluctuation along the field line is on average located on the LFS, just above the midplane, the average value of the fluctuation being $\left\langle\delta n_{\max } / n_{\max }\right\rangle=0.33$ and 0.3 for simulations $\mathrm{A}$ and $\mathrm{B}$, respectively. The parallel size is $\sim 800$ (700) times larger than the poloidal size, corresponding to $35 \%$ (26\%) of the connection length, for simulation A (B). The average values of the pre-sheath density drop are $\left\langle\sigma_{l}\right\rangle=0.32$ and 0.22 for simulations $\mathrm{A}$ and $\mathrm{B}$, respectively, while the theoretical prediction for the sheath limited case is 0.6 [36]. As shown in figure 5 , where the joint probability of the pre-sheath density drop at the two limiter plates is displayed, the blobs disconnect from the bottom limiter plate for high resistivity 

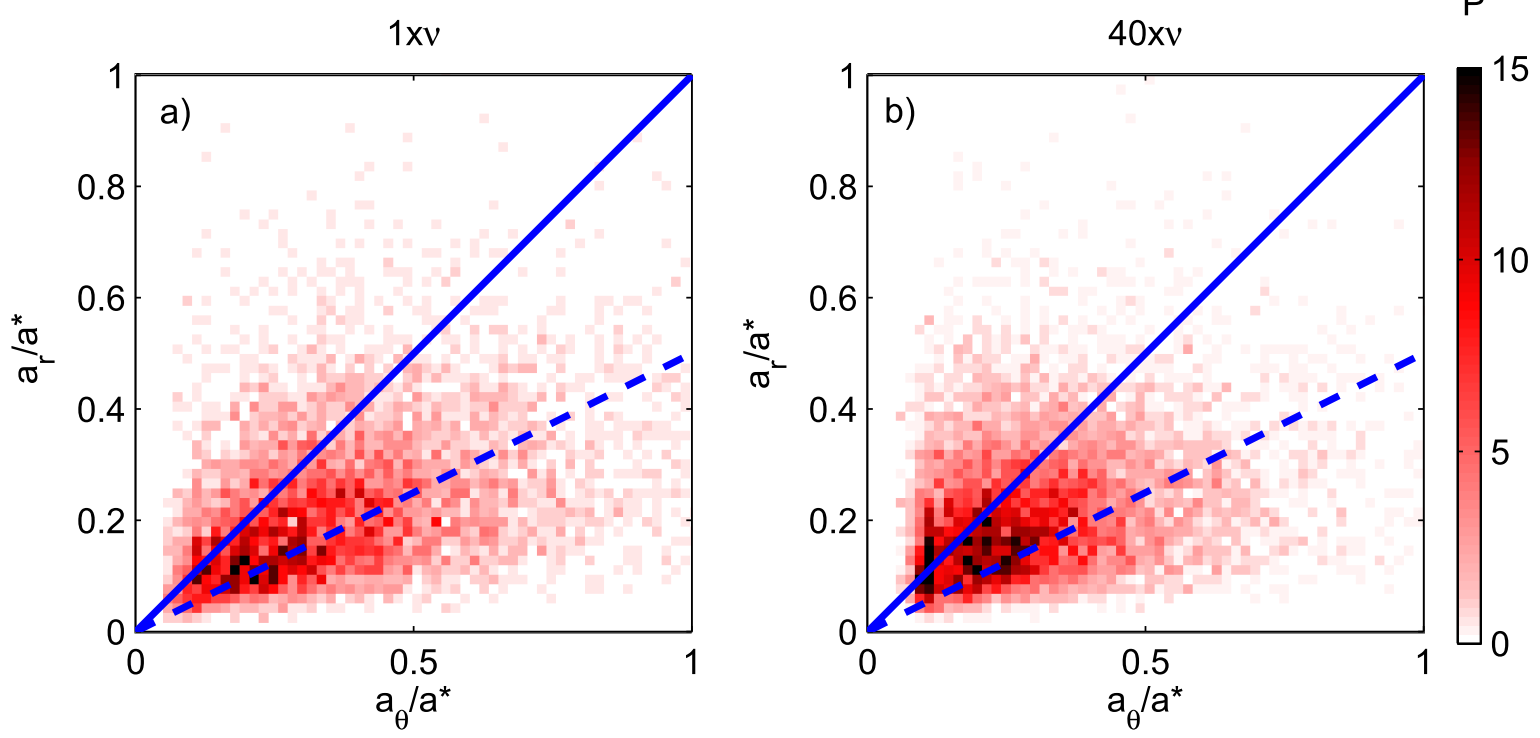

Figure 4. Joint probability distribution $P$ of the poloidal and radial normalized blob size $a_{\theta} / a^{*}, a_{r} / a^{*}$ for simulations A (a) and B (b). The lines $a_{r}=a_{\theta}$ (solid) and $a_{r}=a_{\theta} / 2$ (dashed) are plotted to guide the eye.
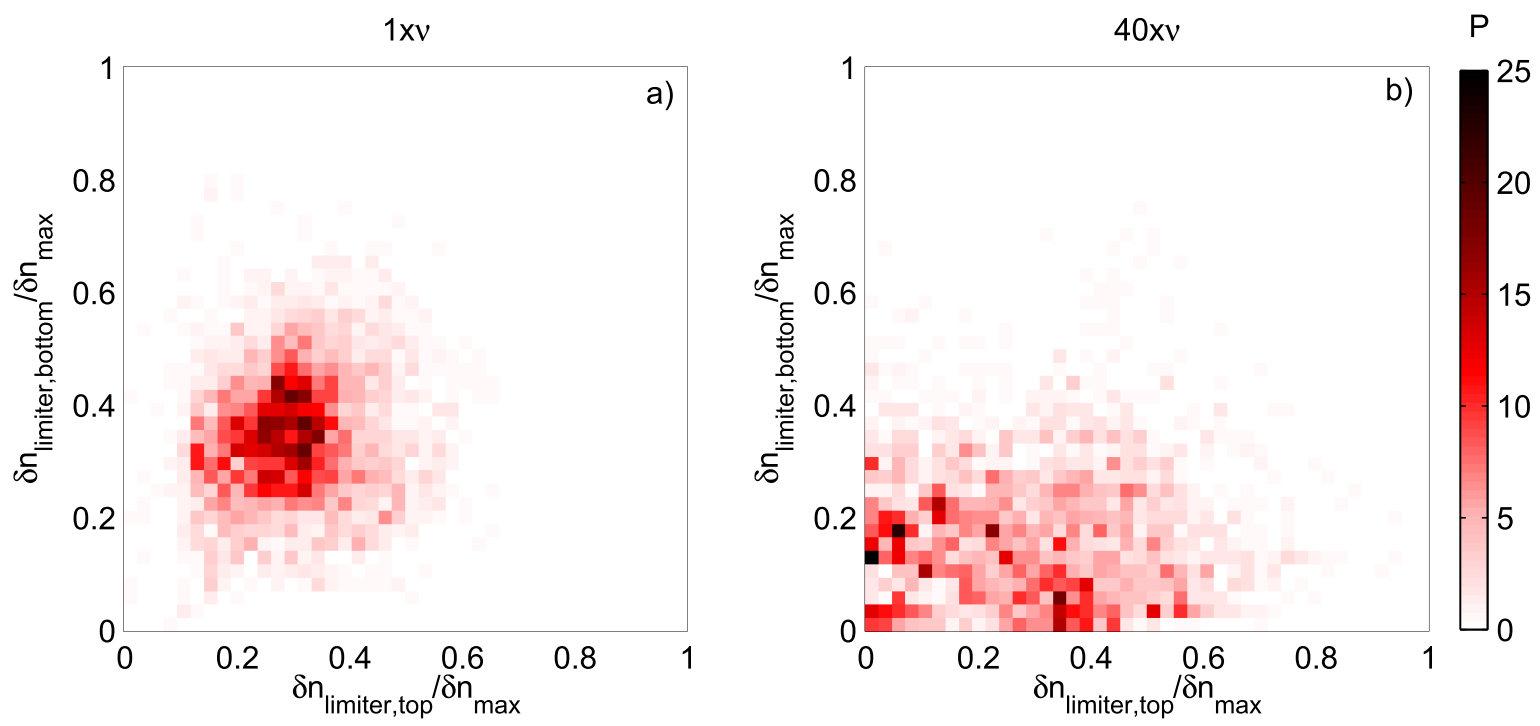

Figure 5. Joint probability distribution $P$ of pre-sheath density drop along the field line $\sigma_{l}=\delta n_{\text {limiter }} / \delta n_{\max }$ on the two sides of the limiter. The values of $\sigma_{l}$ are displayed for simulations A (a) and B (b) respectively.

(simulation B) while remaining partly attached to the top one. In fact, if we arbitrarily consider a blob detached when $\sigma_{l}<0.2$, for simulation B the $66 \%$ of the blobs results detached from the bottom plate and the $36 \%$ of them are detached from the top one, the average values of the presheath density drop at the two limiter plates being $\left\langle\sigma_{l, \text { bottom }}\right\rangle=0.16$ and $\left\langle\sigma_{l, \text { top }}\right\rangle=0.28$.

\section{Cross correlation analysis}

While in the GBS simulations all physical quantities are accessible for the desired time at any point of the 3D domain, experimentally in the tokamak SOL only a number of measurements at fixed locations is available, often with different diagnostics. Typically, fluctuations measurements are carried out at the target (limiter or divertor) using embedded Langmuir probes, while at the LFS one has to rely on gas puff imaging diagnostics [3], reciprocating Langmuir probes [4], or fast framing visible cameras [5]. Cross correlation analysis might be then one of the most powerful and reliable methods to relate target and upstream blob measurements, as done e.g. in [37]. To give some insight on whether this technique provides valuable results, we perform a cross correlation analysis on the GBS simulations and we compare the results with the ones obtained with the blob detection technique.

We compute the cross correlation between the density time trace at a fixed point at the outer midplane $\left(r_{u, 0}, \theta_{0}=\right.$ $\left.0, \phi_{0}=0\right)$ and the density time traces at all the other points in the $3 \mathrm{D}$ domain $\left(r_{u}, \theta, \phi\right)$, where $\phi$ is the toroidal angle. This is 

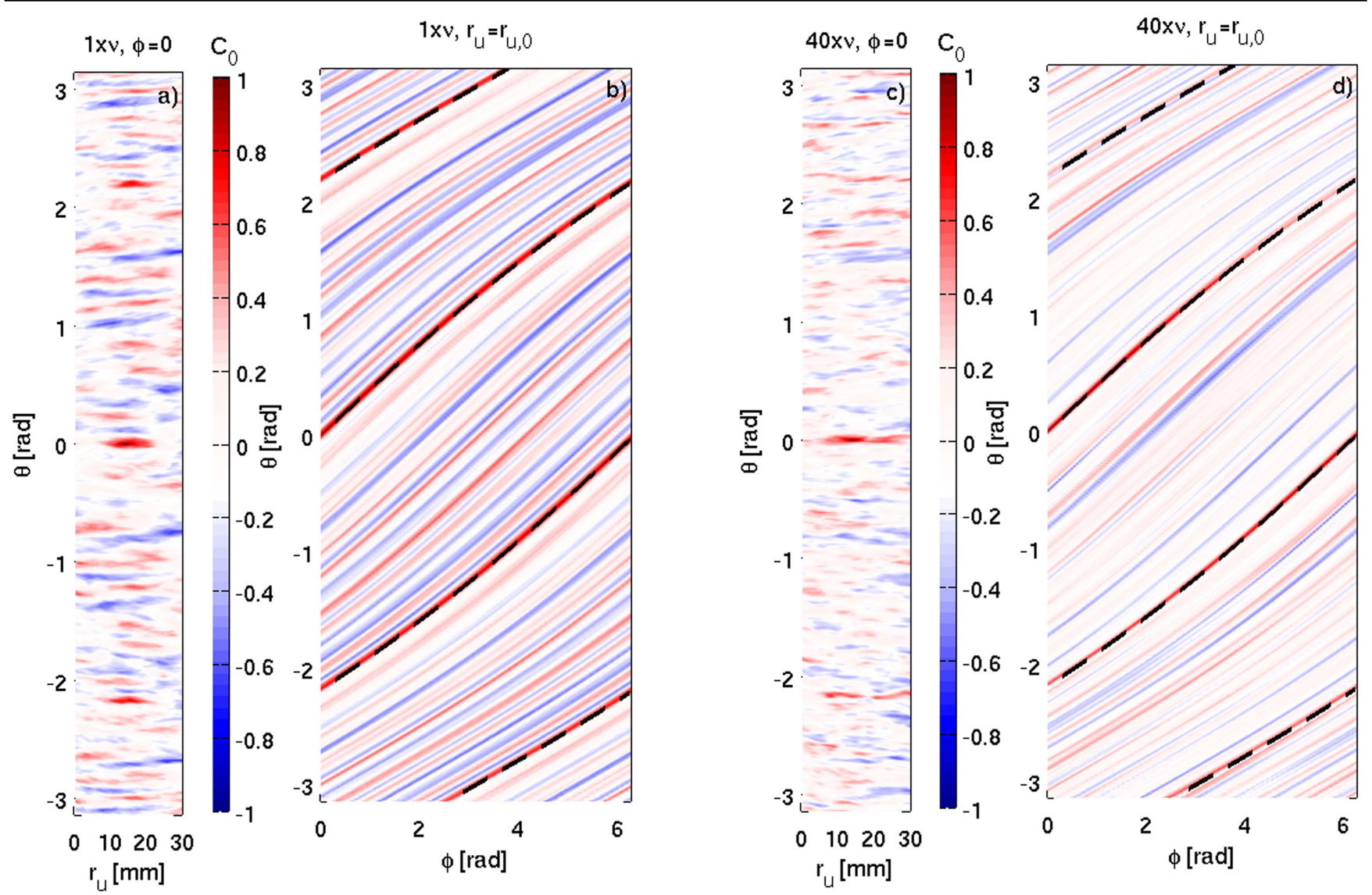

Figure 6. Correlation amplitude at zero time lag $C_{0}$ between a point at the outer mid plane $\left(r_{u, 0}=14 \mathrm{~mm}, \theta=0, \phi=0\right)$ and all the other points in the poloidal section defined by $\phi=0$ (a), (c) and on the flux surface $r_{u}=r_{u, 0}$ (b), (d), for simulation A (a), (b) and B (c), (d). The field line passing through the reference point $\left(r_{u, 0}, 0,0\right)$ is plotted with a black dashed line in $(\mathrm{b}),(\mathrm{d})$.

done for six different values of $r_{u, 0}$ and a typical result is shown in figure 6 , where the amplitude of the correlation function at zero time lag $C_{0}$ is plotted for each point in the poloidal plane and on a flux surface respectively (in the GBS geometry, a flux surface coincides with an $r_{u}=$ const surface). The correlation clearly peaks along the field line passing through the reference point $\left(r_{u, 0}, 0,0\right)$, plotted with a black dashed line in figures 6(b), (d). In figures 6(a), (c) such field line intersects again the poloidal plane $\phi=0$, where two additional peaks of correlation are visible $(q=3.2$ in the simulation).

The profiles of $C_{0}$ along the field line are shown in figure 7 for simulations $\mathrm{A}$ and $\mathrm{B}$, where $C_{0}$ is plotted along the field lines for the six reference points at the outer mid plane. The time lag of maximum of correlation $\tau_{\text {lag }}^{\max }$ is always shorter than $7 \mu \mathrm{s} \sim 0.04 L / c_{s 0}$ along the field line, where $L$ is the connection length. This evidence supports the picture of a blob emerging all along the whole field line from the beginning, rather than being generated mostly on the LFS and progressively elongating towards the limiter.

For simulation $\mathrm{A}, C_{0}>0.5$ all along the field line from limiter to limiter, and the correlation increases moving away from the LCFS, as shown in figure 7. This indicates that the turbulent fluctuations in the SOL become more coherent when moving away from the LCFS, i.e. that the transport in the far

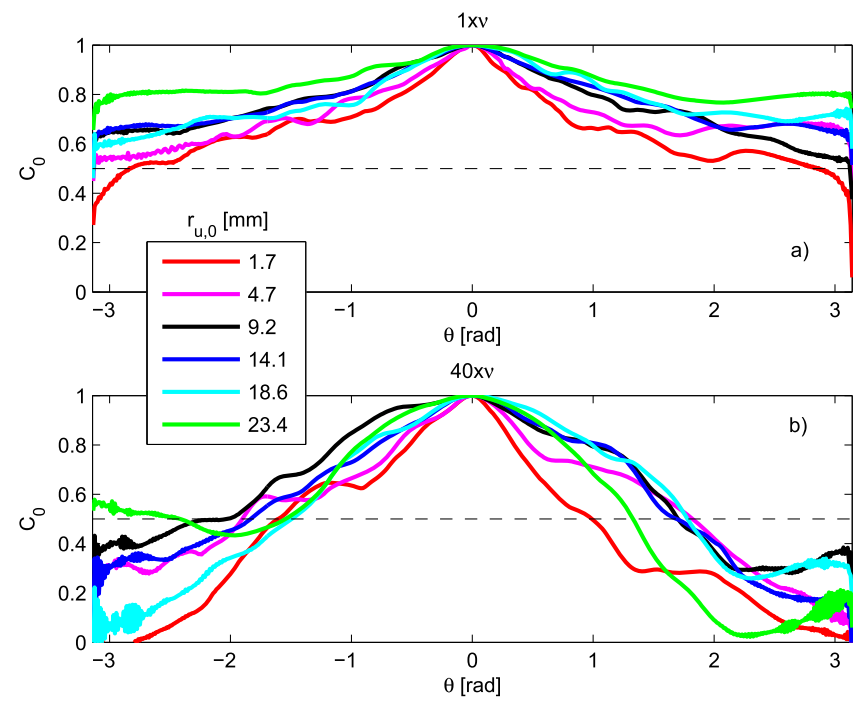

Figure 7. Correlation amplitude at zero time lag $C_{0}$ between a point at the outer midplane $\left(r_{u, 0}, \theta=0, \phi=0\right)$ and all the other points along the field line for simulation A (a) and B (b), plotted against the poloidal coordinate $\theta$.

SOL is dominated by blobs. For simulation $\mathrm{B}, C_{0}$ drops below 0.5 moving towards the limiters for all the considered $r_{u, 0}$ values. The limiter plates and the LFS are therefore decorrelated in simulation B. This picture is consistent with the 


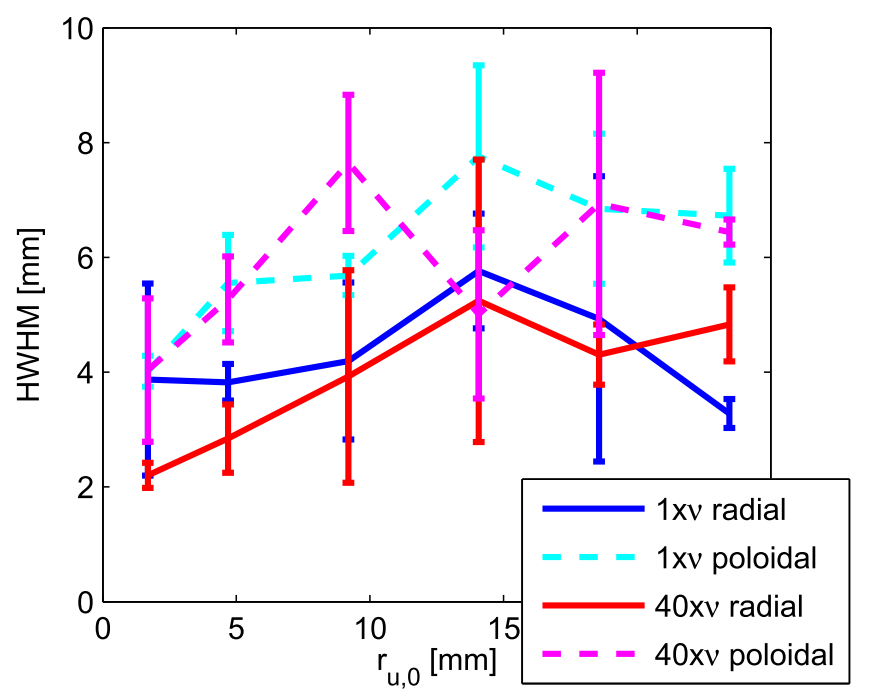

Figure 8. Radial (solid lines) and poloidal (dashed lines) HWHM of the correlation amplitude at zero time lag $C_{0}$ between a point at the outer midplane $\left(r_{u, 0}, \theta=0, \phi=0\right)$ and all the other points on the same poloidal plane for simulation A (blue, cyan) and B (red, magenta). The errorbars are given by the difference between the two HWHMs ('left' and 'right').

result of the analysis of the pre-sheath density drop from the 3D structure of the blobs exposed beforehand, showing that blobs disconnect from the limiter as the resistivity increases (figure 5). From an experimental point of view, this means that cross correlating measurements performed at the limiter and at the LFS would be possible in the low resistivity case (simulation A) but not in the high resistivity one (simulation B).

In the poloidal cross section (figures 6 (a), (c)), the extent of the region for which $C_{0}>0.5$ gives an estimate of the size of the blobs. The variation of the poloidal and radial HWHM of such a region with the radial coordinate $r_{u, 0}$ of the reference point used to compute the correlation are displayed in figure 8 . The blob size estimated in this way varies between 4 and $16 \rho_{s}$, and increases slightly moving radially outwards. These values are compatible with the results from the blob detection algorithm and with what has been found experimentally [3]. Also, the poloidal HWHM is approximately twice the radial HWHM, consistently with the results of the blob detection analysis (figure 4).

\section{Blob velocity}

The detected blob velocities are found to be consistent with the local $\mathbf{E} \times \mathbf{B}$ drift, as shown in figure 9, where the blob radial and poloidal velocities resulting from the tracking algorithm $v_{r}, v_{\theta}$ are plotted against the local components of the $\mathbf{E} \times \mathbf{B}$ drift $v_{E \times B, r}, \quad v_{E \times B, \theta}$, color coded with the absolute value of the blob poloidal position $\theta_{b}$ (averaged over the blob trajectory). The poloidal component of the blob velocity is to be attributed to the background $\mathbf{E} \times \mathbf{B}$ flow, which is mainly poloidal towards the upper limiter. Since the background radial flow is negligible, the blob radial velocity is to be attributed to the $\mathbf{E} \times \mathbf{B}$ drift caused by the electric dipole internal to the blob [1]. Indeed, the effective gravity force associated with $\nabla B$ and magnetic field curvature on the LFS, being the bad curvature region, causes charge separation that polarizes the blob and results in a radially outwards $\mathbf{E} \times \mathbf{B}$ drift. Since blobs are field-aligned, their radial velocity is directed outward also at the HFS. The occurrence of blobs with radial negative velocity, observed both at the HFS and LFS, may be an indication of the interaction of the blobs with the background turbulence. We remark that a small fraction $(<0.5 \%)$ of blobs with negative radial velocity are crossing the LCFS inwards. These blobs are not discarded from our analysis, but they are too rare to have a significant impact on the results.

The average blob velocities are $\left\langle v_{r}\right\rangle \cong 0.016 c_{s 0}=$ $0.15 v^{*}$ and $\left\langle v_{\theta}\right\rangle \cong 0.011 c_{s 0}=0.1 v^{*}$ for simulation A. In simulation $\mathrm{B}$ the higher resistivity causes the blobs to increase their radial velocity of about $10 \%$, while decreasing their poloidal velocity by approximately a factor 2 , being $\left\langle v_{r}\right\rangle \cong 0.02 c_{s 0}=0.18 v^{*}$ and $\left\langle v_{\theta}\right\rangle \cong 0.004 c_{s 0}=0.036 v^{*}$. This is consistent with the substantial decrease in the background $\mathbf{E} \times \mathbf{B}$ flow from simulation A to simulation $\mathrm{B}$, as shown in [30], and with the disconnection of the blobs from the limiter discussed beforehand.

Following [9], the blob radial velocity and poloidal size are normalized $\tilde{v}=v_{r} / v^{*}$ and $\tilde{a}=a_{\theta} / a^{*}$, where $v^{*}$ and $a^{*}$ are defined in equation (1). We compare the results of the blob detection analysis with the scaling

$$
\tilde{v}=\frac{\sqrt{2 \tilde{a}} \delta n / n}{1+2 \sigma_{l} \sqrt{2} \tilde{a}^{5 / 2}}
$$

from [9], where the term due to ion-neutral collisions is here neglected, since neutrals dynamics is not included in these simulations. The inertial limit $\tilde{v}=\sqrt{2 \tilde{a}} \delta n / n$ and the sheath dominated limit $\tilde{v}=\frac{1}{\tilde{a}^{2}} \frac{\delta n / n}{2 \sigma_{l}}$ can be recovered for $\tilde{a} \ll 1$ (or $\left.\sigma_{l}=0\right)$ and $\tilde{a} \gg 1$, respectively. The comparison is carried out in figure 10, where the joint probability distribution of the blob poloidal size versus their radial velocity is plotted for both simulations. The scaling in equation (2) is displayed as a continuous blue line, and its inertial and sheath dominated limits are plotted as a dashed and dot-dashed line, respectively. An overall good agreement between the detected velocities and the scaling predictions is found. Furthermore, the agreement is particularly good with the inertial branch of the scaling. This is consistent with the observation of the blobs being partially disconnected at the limiter $\left(\sigma_{l}=0.32\right.$ for simulation A). The agreement with the inertial branch of the scaling is even better for simulation $\mathrm{B}$, where the blobs detach from the bottom plate of the limiter. The scaling law in equation (2) is derived in the cold ion approximation ( $\left.\tau \equiv T_{i} / T_{e} \ll 1\right)$. In figure 10 , we also compare the detected blob velocities with the scaling in equation (2) corrected for finite ion temperature effects (solid black line), namely

$$
\tilde{v}=\frac{\sqrt{2(1+\tau) \tilde{a}} \delta n / n}{1+\frac{2 \sigma_{l}}{1+\tau} \sqrt{2} \tilde{a}^{5 / 2}},
$$



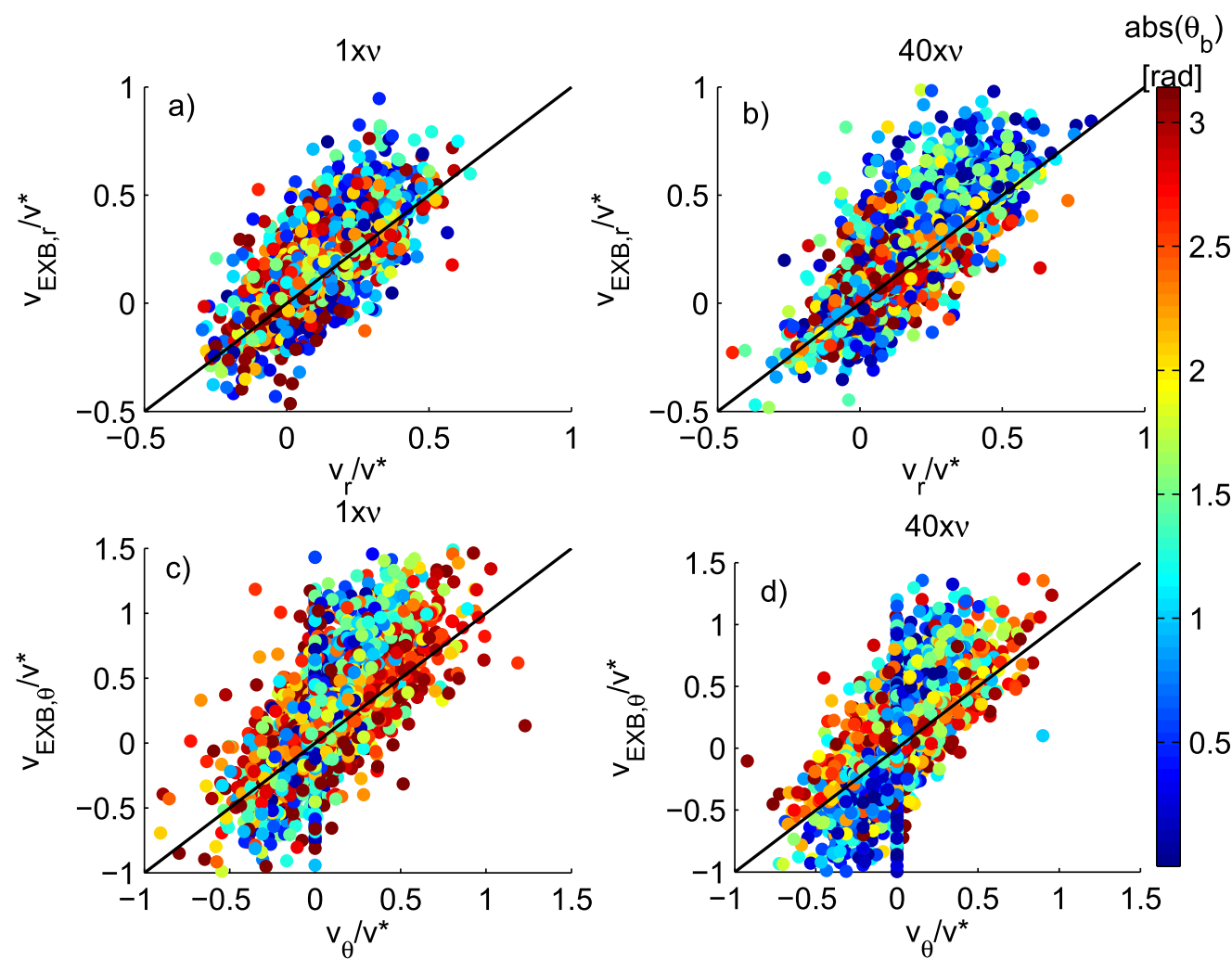

Figure 9. The blob radial and poloidal velocities resulting from the tracking algorithm $v_{r}, v_{\theta}$ are compared with the local components of the $\mathbf{E} \times \mathbf{B}$ drift $v_{E \times B, r}, \quad v_{E \times B, \theta}$, color coded with the absolute value of the blob poloidal position $\theta_{b}$. All quantities are normalized according to equation (1).

$1 \mathrm{xv}$ Total detected blobs $=5902$

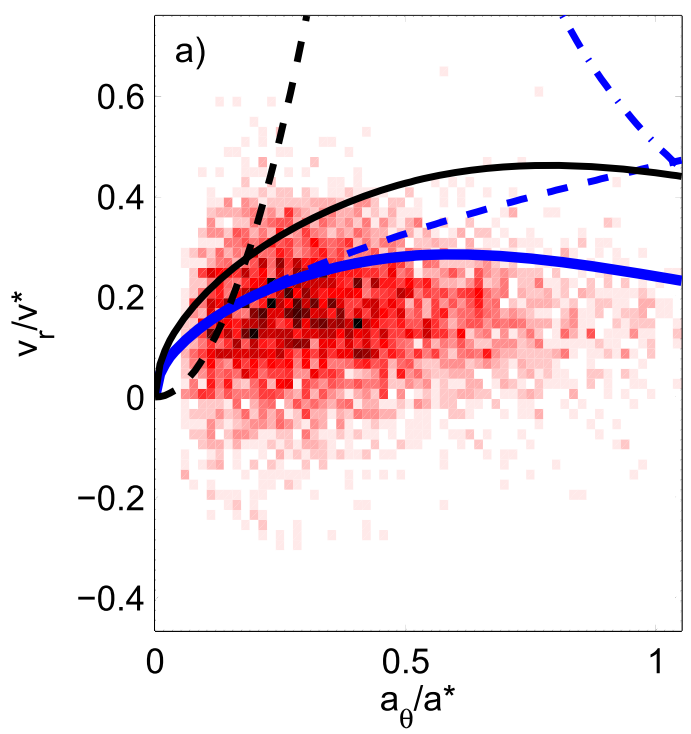

$40 x v$ Total detected blobs $=7611$

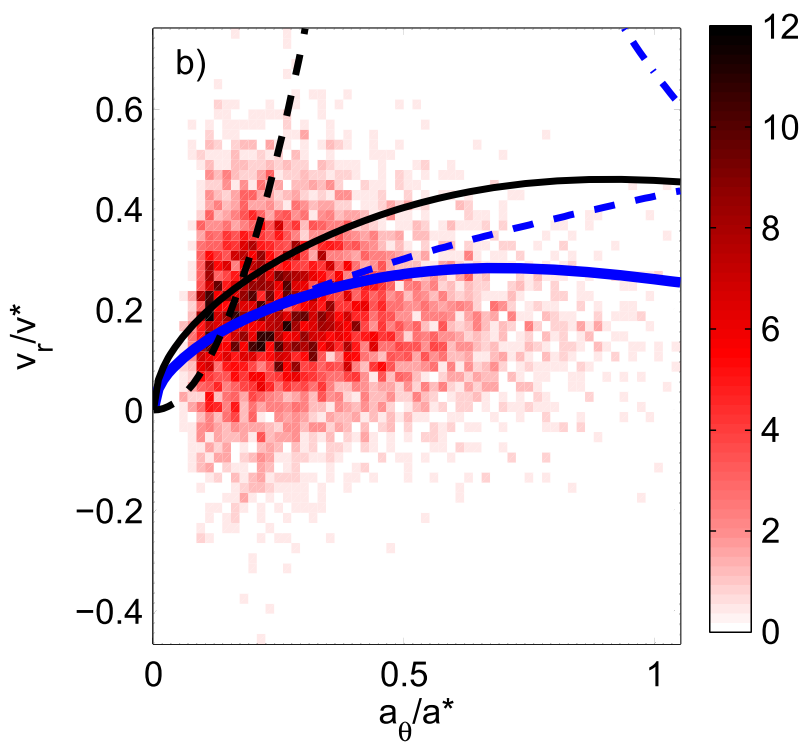

Figure 10. Joint probability distribution $P$ of the normalized blob size in the poloidal plane versus their normalized radial velocity for simulations A (a) and B (b). The scaling in equation (2) (solid blue lines), its inertial limit $\tilde{v}=\sqrt{2 \tilde{a}} \delta n / n$ (dashed blue lines), and the sheath dominated limit $\tilde{v}=\frac{1}{\tilde{a}^{2}} \frac{\delta n / n}{2 \sigma_{l}}$ (dot-dashed blue lines), are also displayed. The scalings in equations (3), (4), including finite $\tau$ effects, are plotted with a solid black line and with a black dashed line, respectively. 

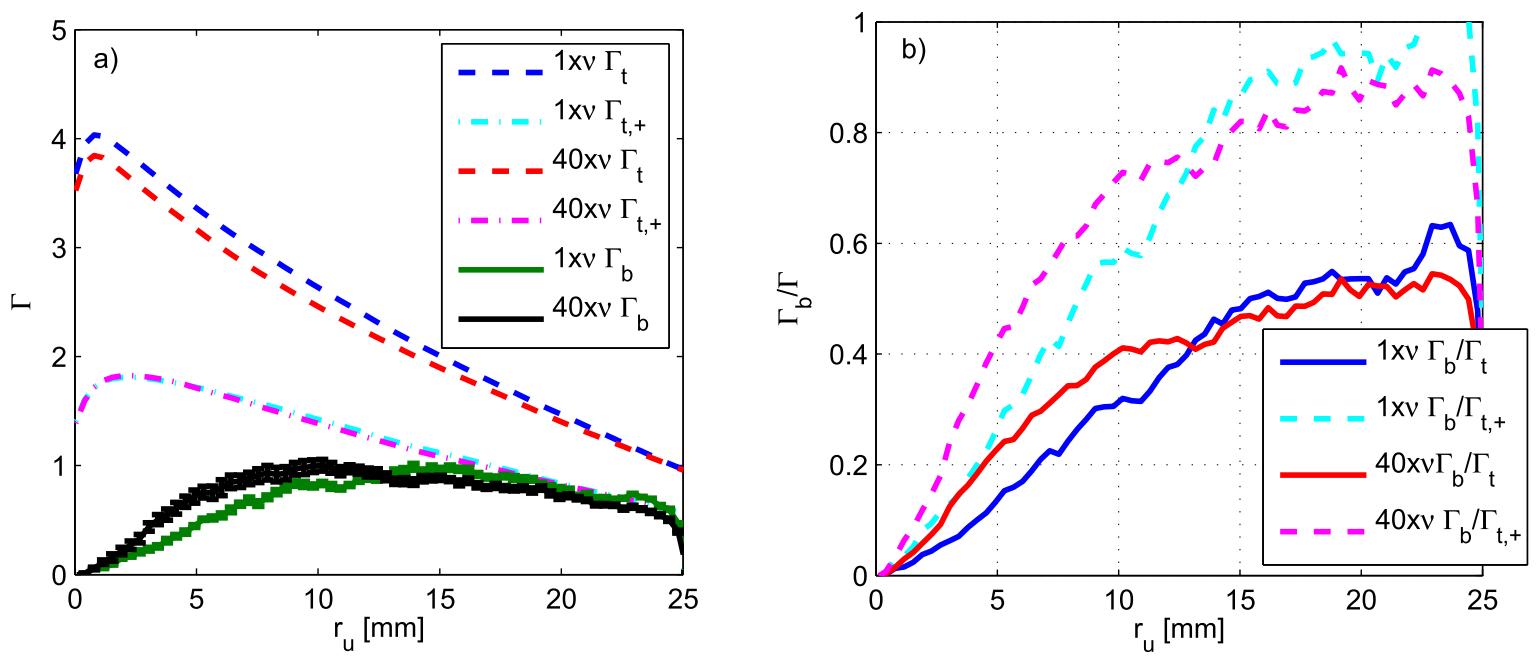

Figure 11. (a) Particle radial fluxes $\Gamma_{b}$ (equation (7)), $\Gamma_{t}$ and $\Gamma_{t,+}$, for both simulations. Errorbars are given by the amount of blobs for which the computation of the HWHMs was not possible. (b) Ratio of blob particle flux over turbulent particle fluxes.

where $\tau=1$. We also consider the scaling law for small blobs including hot ions effect derived in [38] (dashed black line in figure 10), which reads

$$
\tilde{v}=2 \frac{1+\tau}{\tau} \tilde{a}^{2}\left(\frac{8 L^{3} \rho_{s}}{R_{0}^{4}}\right)^{1 / 5},
$$

where we recall $L=2 \pi q R_{0}$. As a result, due to the small size of the detected blobs, no appreciable effects predicted by the scalings in equations (3), (4) are observed in this case. We reserve a detailed investigation of the effects of the finite ion temperature on blob dynamics for future works.

\section{Blob contribution to particle and heat radial fluxes}

Once the blobs are generated, their radial motion is in most cases outwards. We quantify the blob contribution to the radial particle and heat fluxes. In the following, blobs are assumed to have a gaussian profile in the radial and poloidal directions with HWHM $a_{r}, a_{\theta}$ respectively:

$$
\begin{aligned}
\delta n_{b}\left(r_{u}, \theta\right)= & \delta n_{b, \max } \exp \left(-\frac{\left(r_{u}-r_{u, b}\right)^{2}}{2 \sigma_{r}}\right) \\
& \times \exp \left(-\frac{\left[\left(a_{\min }+r_{u, b}\right)\left(\theta-\theta_{b}\right)\right]^{2}}{2 \sigma_{\theta}}\right),
\end{aligned}
$$

where $\sigma_{r}=a_{r} / \sqrt{\ln 2}$ and $\sigma_{\theta}=a_{\theta} / \sqrt{\ln 2}$ are the gaussian widths in the radial and poloidal direction, respectively, $\delta n_{b, \max }$ is the maximum of the density fluctuation inside the blob, located at coordinates $\left(r_{u, b}, \theta_{b}\right)$, and $a_{\min }=0.2 \mathrm{~m}$ is the plasma minor radius. The number of particles advected by each blob is computed as

$$
\begin{aligned}
\delta N_{b} & =\iint \delta n_{b}\left(r_{u}, \theta\right) \mathrm{d} r_{u} \mathrm{~d} \theta=\delta n_{b, \max } 2 \pi \sigma_{r} \sigma_{\theta} \\
& =\delta n_{b, \max } \frac{2 \pi}{\ln 2} a_{r} a_{\theta} .
\end{aligned}
$$

The poloidally and time averaged radial particle flux due to blobs is then computed as

$$
\Gamma_{b}\left(r_{u}\right)=\frac{\sum_{b} \delta n_{b, \max } \frac{2 \pi}{\ln 2} a_{r} a_{\theta}}{2 \pi\left(a_{\min }+r_{u}\right) \Delta t},
$$

where the sum is performed over all the blobs crossing a $r_{u}=$ const line, and $\Delta t=0.55 \mathrm{~ms}$ is the simulation time interval used for all the analysis in this paper. This quantity is compared with the turbulent particle flux $\Gamma_{t}=\left\langle\delta n \delta v_{E \times B, r}\right\rangle$ where the brackets mean average over time and poloidal direction. We remark that the turbulent flux is given by blobs and holes. We consider here only the contribution due to positive density fluctuations $\Gamma_{t,+}=\left\langle\delta n \delta v_{E \times B, r} \mid \delta n>0\right\rangle$, resulting in $\Gamma_{t,+} \sim \Gamma_{t} / 2$. We compare $\Gamma_{b}$ to $\Gamma_{t,+}$ in figure 11(a), where the fluxes $\Gamma_{t}, \Gamma_{t,+}$ and $\Gamma_{b}$ are plotted, for both simulations. The error bars are given by the amount of blobs for which the computation of the HWHMs was not possible. As it emerges from figure 11(b), where the ratios $\Gamma_{b} / \Gamma_{t}$ and $\Gamma_{b} / \Gamma_{t,+}$ are displayed, the flux due to blobs contributes only marginally to the turbulent outward flux in the near SOL, while it accounts for up to $100 \%$ of $\Gamma_{t,+}$ in the far SOL for simulation A and $90 \%$ for simulation $\mathrm{B}$.

We assume that the ion and electron temperature inside a blob are spatially uniform. The poloidally and time averaged radial heat flux due to blobs is then given by

$$
q_{b}\left(r_{u}\right)=\frac{\sum_{b} \delta n_{b, \max } \frac{2 \pi}{\ln 2} a_{r} a_{\theta}\left(T_{e, b}+T_{i, b}\right)}{2 \pi\left(a_{\min }+r_{u}\right) \Delta t}
$$

where $T_{i, b}$ and $T_{e, b}$ are computed as the average ion and electron temperature inside the blob, respectively. The shape of the radial profile of the heat flux associated with the blobs $q_{b}\left(r_{u}\right)$ is qualitatively the same as for the particle flux $\Gamma_{b}\left(r_{u}\right)$. In figure 12 we compare this flux with the turbulent radial heat flux $q_{t}=\left\langle\delta p \delta v_{E \times B, r}\right\rangle$ and with the part of the latter due only to positive pressure fluctuations $q_{t,+}=\left\langle\left.\delta p \delta v_{E \times B, r}\right|_{\delta p>0}\right\rangle$. Similarly to the radial particle flux, we find that the blobs do not contribute substantially to the radial heat flux in the near 

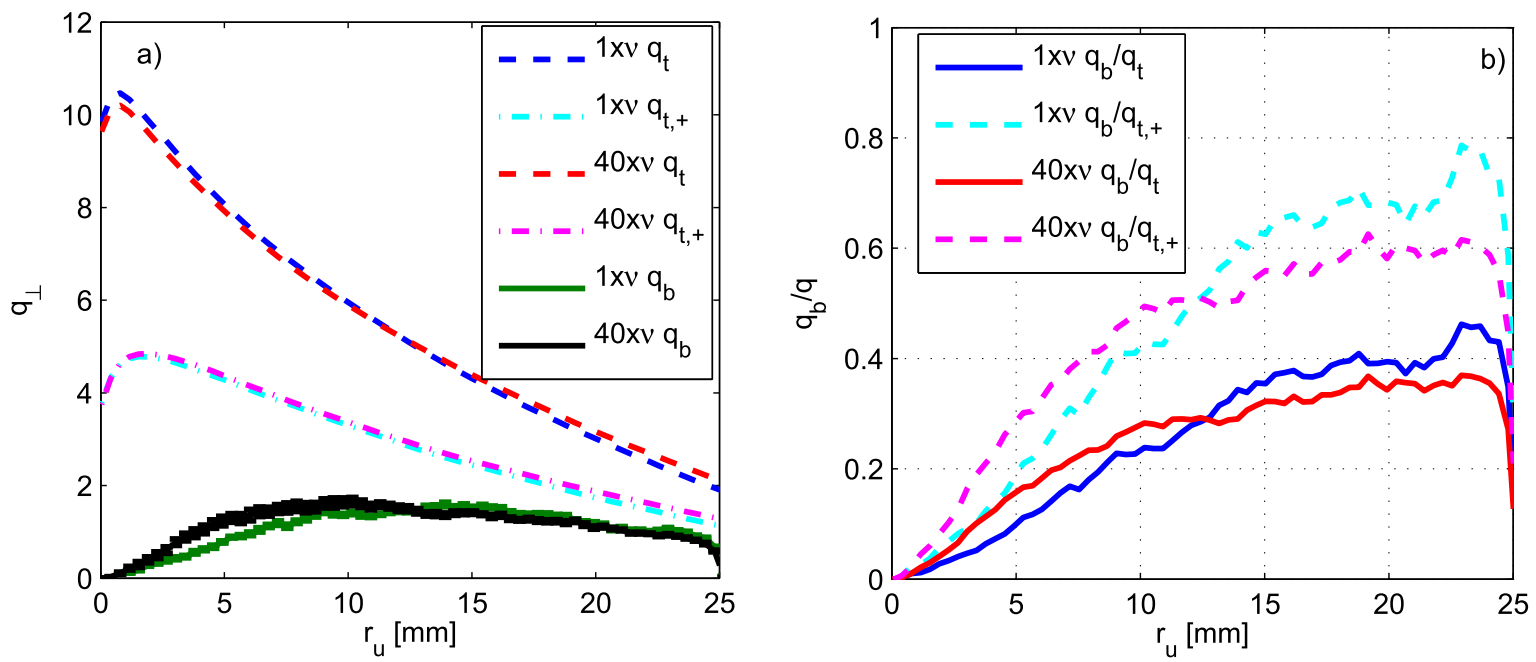

Figure 12. (a) Heat radial fluxes $q_{b}$ (equation (8)), $q_{t}$ and $q_{t,+}$, for both simulations. Errorbars are given by the amount of blobs for which the computation of the HWHMs was not possible. (b) Ratio of blob heat flux over turbulent heat fluxes.

SOL, while they can contribute up to $70 \%(60 \%)$ of the turbulent flux in the far SOL for simulation A (B), respectively.

\section{Conclusions}

A blob detection and tracking algorithm is applied for the first time to 3D full-turbulence simulations of the SOL. We consider an inboard-limited plasma in TCV. The blobs are found to be more elongated poloidally than radially and to be partially disconnected from the limiter. The velocities resulting from the blob tracking algorithm are consistent with the local $\mathbf{E} \times \mathbf{B}$ drift, and their radial velocity agrees well with the inertial branch of the scaling presented in [9]. Once they are generated, the blobs radial motion is mainly outwards. They do not contribute substantially to the particle and heat flux in the near SOL, while they are responsible for up to $100 \%$ and $70 \%$ of the turbulent particle and heat flux in the main SOL, respectively. Increasing the resistivity by a factor 40 causes the blob to detach from the lower limiter and the point of maximum birth rate moves closer to the LCFS. Also, the blobs are more likely to born at the LFS in the high resistivity case. The correlation analysis confirms the results from the blob tracking algorithm, and shows how the blobs are born already all along the whole field line. The application of the same analysis to ongoing simulations including both open and closed field line regions, whose first results are presented in [21, 39], is foreseen to better address the physics at the LCFS and in the near SOL, and in particular the blob generation mechanism. The investigation of the effect of neutrals on blob dynamics is also a topic that can be addressed in future works, thanks to the recent implementation of a kinetic model for neutrals in GBS [40].

\section{Acknowledgments}

The first author gratefully acknowledge useful discussions with $\mathrm{C}$ Theiler, N Fedorczak and O Chellai. The simulations presented herein were carried out in part using the HELIOS supercomputer system at Computational Simulation Centre of International Fusion Energy Research Centre (IFERC-CSC), Aomori, Japan, under the Broader Approach collaboration between Euratom and Japan, implemented by Fusion for Energy and JAEA; in part in part at the Swiss National Supercomputing Centre (CSCS) under Project ID s549. This work has been carried out within the framework of the EUROfusion Consortium and has received funding from the Euratom research and training programme 2014-2018 under grant agreement No 633053. The views and opinions expressed herein do not necessarily reflect those of the European Commission.

\section{References}

[1] Krasheninnikov S I 2008 J. Plasma Phys. 74 679-717

[2] D'Ippolito D A et al 2011 Phys. Plasmas 18060501

[3] Zweben S J et al 2015 Nucl. Fusion 55093035

[4] Carallero D et al 2015 Phys. Rev. Lett. 115215002

[5] Kirk A et al 2016 Plasma Phys. Control. Fusion 58085008

[6] Banerjee S et al 2012 Nucl. Fusion 52123016

[7] Mueller S H et al 2006 Phys. Plasmas 13100701

[8] Mueller S H et al 2009 Plasma Phys. Control. Fusion 51 055020

[9] Theiler C et al 2009 Phys. Rev. Lett. 103065001

[10] Manz P et al 2011 Phys. Rev. Lett. 107195004

[11] Katz N et al 2008 Phys. Rev. Lett. 101015033

[12] Carter T A 2006 Phys. Plasmas 1310701

[13] Windish T et al 2006 Phys. Plasmas 13122303

[14] D'Ippolito D A et al 2003 Phys. Plasmas 104029

[15] Walkden N et al 2016 Plasma Phys. Control. Fusion 58 115010

[16] Riva F et al 2016 Plasma Phys. Control. Fusion 58044005

[17] Easy L et al 2014 Phys. Plasmas 21122515

[18] Agostini C et al 2011 Nucl. Fusion 51053020

[19] Myra J R et al 2013 Nucl. Fusion 53073013

[20] Ricci P et al 2012 Plasma Phys. Control. Fusion 54124047

[21] Halpern F D et al 2016 J. Comput. Phys. 315 388-408

[22] Jovanovic D et al 2008 Phys. Plasmas 15112305

[23] Mosetto A et al 2015 Phys. Plasmas 22012308 
[24] Loizu J et al 2012 Phys. Plasmas 19122307

[25] Myra J R et al 2006 Phys. Plasmas 13112502

[26] Hofmann F and Tonetti G 1988 Nucl. Fusion 281871

[27] Garcia O E et al 2007 Plasma Phys. Control. Fusion 49 B47-57

[28] Kube R et al 2016 Plasma Phys. Control. Fusion 58054001

[29] Easy L et al 2016 Phys. Plasmas 23012512

[30] Nespoli F et al 2016 Nucl. Mater. Energy (https://doi.org/ 10.1016/j.nme.2016.10.019)

[31] Nespoli F et al 2015 J. Nucl. Mater. 463 393-6
[32] Kocan M et al 2015 Nucl. Fusion 55033019

[33] Mosetto A et al 2013 Phys. Plasmas 20092308

[34] Yu G Q and Krasheninnikov S I 2003 Phys. Plasmas 104413

[35] Aydemir A Y 2005 Phys. Plasmas 12062503

[36] Chen F F and Chang J P 2003 Lecture Notes on Principles of Plasma Processing (Dordrecht/New York: Kluwer/Plenium)

[37] Grulke O et al 2014 Nucl. Fusion 54043012

[38] Manz P et al 2013 Phys. Plasmas 20102307

[39] Halpern F D and Ricci P 2017 Nucl. Fusion 57034001

[40] Wersal C and Ricci P 2015 Nucl. Fusion 55123014 